\title{
La hostilidad: Sus efectos en la salud y medición psicométrica en Latinoamérica
}

Manolete Moscoso

Se describe la importancia del estudio de la hostilidad y sus efectos en la salud, haciendo referencia al fenómeno del estrés y sus conexiones con el sistema inmune. Se presenta un análisis de aspectos conceptuales y teóricos de la hostilidad. Se reporta la validez de construcción del Inventario Multicultural Latinoamericano de la Hostilidad (Imulho). En base a una muestra multicultural, se realizó un análisis factorial de componentes principales con rotaciones promax con el fin de determinar el nivel de correspondencia de la estructura factorial en el instrumento. Los resultados reportan que el Imulho demuestra un alto nivel de validez de construcción y consistencia interna, reflejada en su estructura factorial y coeficientes alpha.

Hostilidad / Estrés / Psiconeuroinmunología / Validez de construcción / Salud

\section{Hostility: Its impact on health and psychometric measuring in Latin America}

The article describes the importance of research on hostility and its consequences on health and the connections between emotions and immune system. We present a view of the conceptual and theoretical framework of hostility. One of the main goals of this study was to report the construct validity of the Multicultural Latin American Hostility Inventory. Responses to the items, were factored in separate principal factor analysis with promax rotations to determine the level of correspondence of factor structure in the instrument. This inventory presents with empirical evidence that demonstrates a high degree of construct validity and internal consistency as shown by its factorial structure and alpha correlations.

Hostility / Stress / Psychoneuroimmunology / Construct Validity / Health 


\section{INTRODUCCIÓN}

Estudios de investigación llevados a cabo en las dos últimas décadas muestran una correlación elevada entre estados emocionales negativos, como la hostilidad y la pérdida de la salud. El nuevo campo interdisciplinario de la psiconeuroinmunología nos ofrece un magnífico marco de referencia para el estudio de las respuestas fisiológicas que se producen en el sistema nervioso, sistema endocrino y sistema inmune, los cuales son afectados directamente como consecuencia de la expresión de emociones negativas como la hostilidad.

El propósito de nuestro estudio tiene varios frentes: en primer lugar, establecer una clara distinción del concepto de hostilidad en relación con otros dos conceptos íntimamente relacionados: la cólera y la agresión. En segundo lugar, mejorar nuestro entendimiento de la hostilidad como una reacción emocional, y, al mismo tiempo, como un patrón de conducta dentro del proceso de estrés y afrontamiento. Asimismo, hacer un breve análisis y revisión de la posible interacción de la hostilidad con el sistema immune. Finalmente, presentar la validez de construcción de un inventario de hostilidad con características multiculturales que permitan una adecuada medición psicométrica de esta emoción en el contexto latinoamericano.

\section{DEFINICIÓN DE LA HOSTILIDAD}

A pesar de la extensa cantidad de estudios científicos que reportan acerca del impacto negativo de la hostilidad sobre la salud y el bienestar psicológico de las personas, la definición de este término es bastante ambiguo y muchas veces confuso (Biaggio, Supplee \& Curtis, 1981; Spielberger \& Moscoso, 1995; Moscoso y Pérez-Nieto, 2003; Spielberger, Moscoso \& Brunner, 2005). Debido sustancialmente a que los términos de la hostilidad, cólera y agresión son usados de manera frecuente en forma intercambiable, los referimos en forma colectiva como el Síndrome AHA (Anger, Hostility, Aggression).

En este sentido, es útil presentar la definición operacional de los componentes del Síndrome AHA, propuesta por Spielberger en 1983:

El concepto de la cólera se refiere a un estado emocional, el cual consiste de sentimientos que varían en intensidad, desde una ligera irritación o molestia hasta furia intensa y rabia. A pesar de que la hostilidad usualmente incluye sentimientos de cólera, este concepto lleva la connotación de un conjunto de actitudes complejas, las cuales motivan conductas agresivas dirigidas hacia la destrucción de objetos o de daño físico hacia otras personas. Mientras que la cólera y hostilidad se refieren a sentimientos y actitudes, el concepto de agresión por lo general implica una conducta punitiva o destructiva hacia otras personas u objetos (p. 160). 
Esta distinción operacional estableció los fundamentos teóricos para el estudio de la cólera, la hostilidad y la agresión, y desarrolló las bases para la construcción del State-Trait Anger Inventory (STAS) elaborado por Spielberger y colaboradores (1983). Al mismo tiempo, nos permitió entender que la hostilidad es una reacción emotiva que incluye sentimientos de cólera, y, a la vez, mantiene una connotación actitudinal que la convierte, además, en un patrón de conducta orientada hacia la violencia. Esta connotación actitudinal está formada en base a un sistema de creencias negativas sobre la naturaleza humana y la sociedad. La personalidad hostil se caracteriza por presentar atribuciones "de ser victima" debido a una sociedad injusta, mezquina, egoísta, deshonesta y que, en esencia, representa una amenaza a su proceso de adaptación.

\section{LA HOSTILIDAD COMO REACCIÓN EMOCIONAL AL ESTRÉS}

El estrés constituye una parte integral de la textura natural de la vida. Este puede ser definido como un proceso que incluye interacciones entre el individuo y su medio ambiente, durante el cual los estresores están íntimamente conectados con reacciones emocionales de los individuos que así lo perciben. En este sentido, el afrontamiento al estrés es un requisito básico dentro de la experiencia humana, en la cual la hostilidad tiene un rol central y se presenta como una forma emotiva de reac- ción frente a la percepción de amenaza originada por tal estrés. La figura 1 nos permite tener un mejor entendimiento de la hostilidad como respuesta al fenómeno del estrés.

Resultados de estudios de investigación publicados en las dos últimas décadas han permitido reconocer que la relación entre estrés y enfermedad es en gran medida un proceso complejo y difícil de evaluar. Existe un conjunto de variables disposicionales, estilos de afrontamiento (Moscoso, 1992, 1995), percepción evaluación cognitiva (Spielberger \& Moscoso, 1996) y recursos personales a disposición, los cuales cumplen un rol importante en este complejo proceso (Cohen et al., 1982; Elliott \& Eisdorfer, 1982; Anson et al.,1993). Por ejemplo, está claramente establecido que el Tipo Conductual A genera una mayor reactividad cardiaca bajo estados de estrés, lo cual tiene serias implicancias en desórdenes cardiovasculares (Matthews et al., 1986; Moscoso, 1989; Moscoso, 1995). Los niveles de hostilidad observados en Patrón Conductual A han sido reconocidos como un factor de riesgo en este tipo de enfermedades. La hostilidad, como rasgo o temperamento, es también un buen ejemplo de las diferencias individuales a respuestas de estresores, que dependen de las características particulares del individuo de percibir, afrontar y controlar las condiciones de peligro (Lazarus \& Folkman, 1984; Pearlin, 1991). 


\section{Figura 1}

Un modelo conceptual de estrés, afrontamiento y hostilidad, especificando la relación entre estos constructos y mediadores externos, internos, evaluación cognitiva de amenaza (Appraisal)

\section{Estrés, afrontamiento y hostilidad}

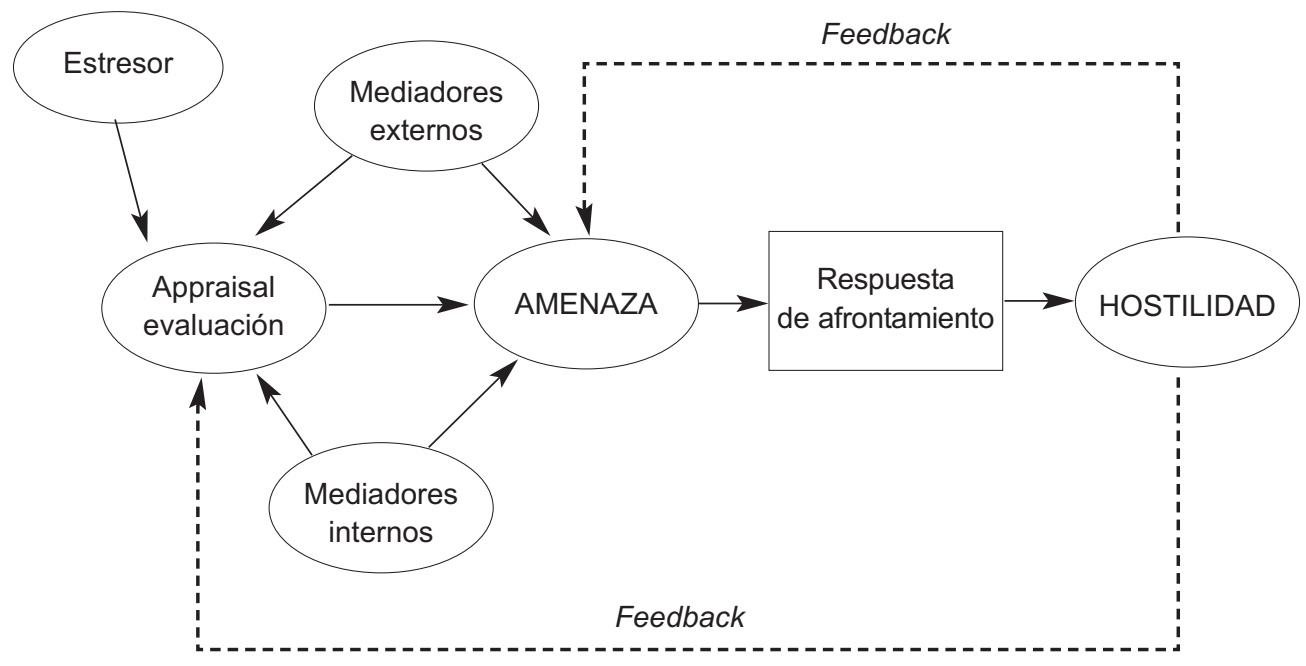

La percepción de control sobre el estresor es otro poderoso factor mediador de respuesta a eventos estresantes observada en personas hostiles. Los estresores evaluados cognitivamente como negativos, incontrolables o ambiguos, son por lo general percibidos como de mayor peligro (Fleming et al., 1987). A diferencia del pobre nivel de afrontamiento que presentan las personas con niveles altos de hostilidad, el afrontamiento efectivo del estrés juega un rol muy importante en la promoción de la salud, prevención de enfermedades y la recuperación rápida y eficaz (Kessler \& Wortman, 1988).
La actitud y conducta que un individuo adopta para enfrentar el estrés es un importante mediador en la relación salud-enfermedad.

En este sentido, los estilos de afrontamiento pueden influenciar en los niveles hormonales o afectar el funcionamiento del sistema inmune (Jacobs et al.,1985; Kielcolt-Glasser et al.,1988). Por ejemplo, el afrontamiento proactivo, que incluye el optimismo, tiene una elevada correlación con reacciones fisiológicas óptimas y saludables (Carver et al., 1989; Scheier \& Carver, 1987; Peterson \& Seligman, 1987; Taylor et al., 1992; Carver et al., 1993). 
EfECTOS DEL ESTRÉS SOBRE EL SISTEMA INMUNE

Un buen número de estudios de investigación se han interesado en observar la interacción entre estrés, funciones endocrinas y sistema inmune. Estos estudios de carácter multidisciplinario han permitido entender que ciertos procesos conductuales son capaces de influenciar funciones del sistema inmune $\mathrm{y}$, por otro lado, el estado inmunológico de un organismo tiene influencia significativa sobre la conducta. Esta línea de investigación es una promesa en el campo de la psicología de la salud y de la medicina integral. Los resultados de estos estudios nos permiten entender que el sistema inmune y el sistema nervioso, considerados como dos de los más complejos sistemas en el mantenimiento de la homeostasis, representan un mecanismo integrado que contribuye a la adaptación del individuo (Ader \& Cohen, 1985).

El sistema inmune es entendido como un mecanismo de vigilancia y defensa cuya responsabilidad es combatir enfermedades causadas por microorganismos y otros agentes externos. Estudios de interacciones entre procesos inmunológicos y psicológicos mediados por el estrés, como en el caso de la hostilidad, son un reto para poder explicar muchas de las conexiones psicofisiológicas entre conducta y enfermedad (Ader, 1983).
Estudios realizados en animales nos permiten observar que estresores creados en el laboratorio pueden suprimir las respuestas de defensa del sistema inmune a través de la reducción de linfocitos (Ader, 1981; Ader \& Cohen, 1991; Ader et al., 1990). Del mismo modo, datos de estudios empíricos con seres humanos indican que eventos estresantes como la cirugía, los exámenes académicos y excesivos periodos de vigilia, están asociados con reducciones en el nivel de concentración de linfocitos y células $\mathrm{T}$ en el sistema inmune (Jemmott \& Locke, 1984; Glaser et al., 1987). Evidencia más directa en términos de reducción de linfocitos en el sistema inmune mediado por estrés ha sido reportada por Dorian et al. (1982).

El desarrollo de la psiconeuroinmunología como una nueva ciencia interdisciplinaria es de gran beneficio en el campo de la medicina y la psicología, particularmente dentro del área interdisciplinaria de la medicina integral. Este nuevo avance permite clarificar, en cierta medida, las complejas interacciones entre variables psicológicas y el paradigma salud-enfermedad. Psicólogos de la salud trabajando conjuntamente con inmunólogos, endocrinólogos y neurólogos tienen la misión de responder preguntas originadas por la mediación de las emociones y el estrés sobre el sistema inmune. Así mismo, la psiconeuroinmunología representa un cambio significativo en términos de pensamiento y en la forma de concep- 
tualizar los procesos inmunológicos, ya que tradicionalmente este sistema era entendido de manera autónoma e independiente, y sin posibilidades de relación con variables psicológicas o emocionales (Kemeny et al., 1987).

La psiconeuroinmunología ha abierto una gran puerta hacia nuevas posibilidades en los campos de la medicina integral y psicología de la salud, y a su vez, ha proveído los lineamientos necesarios para la utilización de estrategias de intervención psicológica y conductual dirigidas a mejorar el funcionamiento inmunológico a través de la modificación en la percepción y la evaluación de estresores.

De acuerdo con estudios recientes de investigación, el estrés y las reacciones emocionales de hostilidad y ansiedad han sido consideradas factores mediadores de funciones inmunológicas; por ejemplo en pacientes infectados con el VIH (Moscoso, 1997; Mays \& Moscoso, 1995). Asimismo, el estrés percibido por pacientes con enfermedades que representan una amenaza a sus vidas, es considerado como un factor mediador en el deterioro de la enfermedad (Solomon \& Temoshok, 1987; Glaser \& Kiecolt-Glaser, 1987; Kiecolt-Glaser \& Glaser, 1988; Laperriere et al., 1989; Antoni et al., 1990).

Recientemente, investigadores y profesionales de la salud han llegado a entender que las enfermedades cardiovasculares se encuentran íntimamente relacionadas con enfermedades infla- matorias causadas, en gran medida, por el sistema inmune y el sistema endocrino. En este sentido, Suárez y colaboradores se encuentran realizando una serie de estudios en la universidad de Duke, en Carolina del Norte, acerca de la hostilidad y estados depresivos y sus efectos sobre el sistema inmune y endocrino. Estos autores consideran que la hostilidad, así como también los estados depresivos pudieran tener algún nivel de influencia sobre ciertas enfermedades cardiovasculares. A la fecha, este equipo de investigadores reporta que niveles altos de hostilidad elevan de manera significativa las concentraciones de plasma Interleukin-6 ( IL-6 ), que es una marca inmunológica asociada con inflamaciones (Suárez, 2003; Suárez, et. al., 2003). Este mismo autor describe, en otro estudio, que puntajes elevados en hostilidad están asociados con un aumento de las proteínas Creactivas (C-Reactive Protein), también relacionada con inflamaciones, lo que representa un factor de riesgo en las enfermedades cardiovasculares (Suárez, 2004).

En otro estudio de características similares, Stoney y Engebretson (2000) reportan que la hostilidad está asociada con un aumento en los niveles de catecolaminas, los cuales, a su vez, elevan el nivel de estrés oxidativo y las concentraciones de plasma homocysteine. Este aumento en homocysteine representa un significativo factor de riesgo en las enfermedades cardiovasculares. 
No es menos importante referirnos al estudio de Christensen y colaboradores en el que se reporta un significativo aumento de actividad en células NK (natural killer cells) en individuos que mostraron niveles altos de hostilidad durante un reportaje verbal como parte de este estudio (Christensen et. al., 1996).

\section{MEDICIÓN PSICOMÉTRICA DE LA HOSTILIDAD}

Debido al creciente interés que actualmente existe en evaluar la hostilidad en las áreas de la psicología de la salud y la medicina integral, hace aproximadamente una década iniciamos estudios de investigación con el propósito de construir un inventario que tuviera propiedades psicométricas adecuadas para evaluar la hostilidad en Latinoamérica, utilizando muestras multiculturales de diversos países de América del Sur, México y el Caribe, y analizar la estructura factorial de la prueba en sujetos de habla hispana (Moscoso \& Reheiser, 1996; Moscoso \& Spielberger, 1997, 1998).

Uno de los propósitos de este artículo es reportar la validez de construcción del Inventario Multicultural Latinoamericano de la Hostilidad (Imulho), que propone medir la experiencia y expresión de la hostilidad en diversos grupos de personas de habla hispana. Los ítems del Imulho fueron desarrollados en base al marco teórico y conceptual del StateTrait Anger Scale (STAS), con la inten- ción de evaluar las mismas dimensiones del Estado-Rasgo como sucede con el STAS (Moscoso \& Reheiser, 1996).

\section{MÉTOdo}

\section{Participantes}

La muestra utilizada para el estudio de la validez de construcción y consistencia interna del Imulho está compuesta por un total de 376 participantes (179 mujeres y 197 varones) de 18 países de América del Sur, México y el Caribe, quienes participaron en el 27 Congreso Interamericano de Psicología (SIP) realizado en Caracas, Venezuela, en julio de 1999. Se obtuvo información demográfica de cada sujeto en relación con la edad, la nacionalidad, el género y el nivel de educación. Las edades de los sujetos varían entre 20 y 79 años, siendo el promedio 34 años.

\section{Procedimiento}

La aplicación del instrumento se llevó a cabo en el hotel Hilton de la ciudad de Caracas, sede del congreso. La muestra se alcanzó al ofrecer instrucciones verbales y escritas a todos los participantes. La muestra verbal se obtuvo al final de siete sesiones científicas, solicitándoles a los sujetos que completen la página de información demográfica y que respondan a los 56 ítems del cuestionario titulado "autoevaluación" y que luego hagan comentarios de carácter lingüístico, si fuera 
necesario, al final de la prueba. Para la muestra escrita se repitieron las mismas instrucciones a todos los participantes. Esta se realizó en grupos de 5 a 10 personas en la sala de exposiciones e información del congreso. En ambos casos, todos los participantes firmaron un consentimiento de participación en forma voluntaria; se les ofreció toda la información posible acerca del estudio y se mantuvo el anonimato del participante. Cada sujeto completó el cuestionario y, al final, cada participante tuvo la oportunidad de hacer comentarios en relación con ciertas diferencias o equivalencias lingüísticas de los ítems.

\section{Construcción de ítems del Imulho}

La construcción y la adaptación de los 56 ítems que inicialmente formaron parte del Imulho se llevaron a cabo en el invierno de 1995. La primera fase del proceso de elaboración de estos ítems consideró los siguientes principios metodológicos: 1) El marco teórico y conceptual que sirvió de guía en la construcción de ítems del STAS y del State-Trait Anger Expression Inventory (Spielberger, 1983); 2) La teoría de equivalencia psicométrica y el marco conceptual de respuesta de ítems en medición transcultural (Drasgow \& Hulin, 1987; Hulin, 1987; Sperber, Devellis \& Boehlecke, 1994).

Algunos de los 56 ítems fueron construidos y desarrollados de manera específica para su incorporación en el
Imulho. Estos ítems fueron elaborados en base al marco conceptual y teórico que guió la construcción del STAS. Otro grupo significativo de ítems fue adaptado en base a la equivalencia gramatical y de concepto. Este aspecto es particularmente importante debido al hecho de que el Imulho tiene como propósito ser utilizado con individuos de diferentes culturas latinoamericanas; en este sentido, es vital que los ítems adaptados de la forma original mantengan equivalencia en cuanto a la experiencia del concepto.

Durante el proceso de elaboración de ítems se evitó la traducción de frases idiomáticas. No se incorporaron los ítems de la versión original del STAS que reflejan aspectos idiomáticos en la lengua inglesa; por ejemplo: "I am burned up", "I fly off the handle". La traducción directa de frases idiomáticas es reconocida como una fuente de limitación en el proceso de adaptación de instrumentos psicológicos en investigación transcultural (Brislin, 1970). Finalmente, es importante indicar que algunos ítems del STAS fueron traducidos directamente debido a su fácil adaptación al nuevo instrumento, sin peligro de que pierdan la equivalencia gramatical y la experiencia conceptual de dichos ítems; por ejemplo: "estoy furioso/ I am furious".

La segunda fase en la construcción del Imulho incluye la presentación de los 56 ítems a un grupo de 26 prominentes y experimentados psicólogos de 
diversos países latinoamericanos, quienes cumplieron el rol de jueces, con el propósito de evaluar los ítems cualitativamente sobre la base de los comentarios de carácter lingüístico y adecuación del instrumento en las diversas culturas de América del Sur, México y el Caribe (Moscoso \& Spielberger, 1997, 1998). El Imulho presenta instrucciones específicas para las subescalas de temperamento y reacción de la hostilidad. Estas requieren que el sujeto conteste la totalidad de los ítems sobre la base de una frecuencia de 4 puntos (Casi nunca 1, Algunas veces 2, Frecuentemente 3, Casi siempre 4).

\section{Resultados}

Con el objeto de verificar si las subescalas del Imulho mantienen consistencia interna y una adecuada estructura factorial en la muestra latinoamericana, los ítems fueron expuestos al análisis factorial de componentes principales con rotaciones promax, tanto para mujeres como para varones. Los resultados de estos análisis nos permitieron identificar las escalas de Temperamento y Reacción de la hostilidad, que, a su vez, tienen valores eigen superiores a 1 , en varones y mujeres. Resultados de dichos análisis, incluyendo las tablas que muestran los pesos factoriales de cada uno de los ítems, porcentajes de varianza, coeficientes alpha e intercorrelaciones de ítems, han sido reportados previamente (Moscoso \& Spielberger, 1999a; 1999b; Moscoso, 2000). Las medias, desviaciones estándar y los valores $\mathrm{F}$, tanto para mujeres como para varones, se indican en la siguiente tabla.

Tabla 1

Medias, desviación estándar y coeficientes Alpha del Inventario Multicultural Latinoamericano de la Hostilidad (Imulho) para mujeres y varones, así como los valores $\mathrm{F}$ y el nivel de significación en las diferencias por género

\begin{tabular}{lrrrrrrr}
\hline \multicolumn{7}{c}{ Puntajes de mujeres } & \multicolumn{2}{c}{ Puntajes de varones } \\
\hline Escalas & Media & Des. est. & Alpha & Media & Des. est. & Alpha & F-Value \\
\hline Hostilidad & 20.16 & 5.32 & .86 & 18.78 & 4.73 & .8 & $3.90^{*}$ \\
T/Temp. & 8.53 & 2.86 & .85 & 7.76 & 2.54 & .81 & $4.20^{*}$ \\
T/Reac. & 11.64 & 3.28 & .78 & 11.03 & 3.13 & .77 & 1.94 \\
\hline
\end{tabular}

${ }^{*} \mathrm{p}<.05 ;{ }^{* *} \mathrm{p}<.01 ;{ }^{* * *} \mathrm{p}<.001$ 
Como resultado de este primer análisis factorial, se seleccionaron 10 ítems sobre la base de dos criterios fundamentales: 1) poseer un peso factorial mínimo de .35 en su propio factor y saturaciones insignificantes en los demás factores; 2) considerar los ítems que mantuvieran coherencia conceptual en el factor dominante. Los 10 ítems que actualmente conforman las dos subescalas del Imulho fueron analizados factorialmente. Se decidió utilizar el análisis factorial exploratorio, en lugar del análisis factorial confirmatorio, debido a que el primero facilita un tipo de análisis más conservador (Gorsuch, 1988).

Resultados del análisis factorial de la escala de hostilidad nos permite con- firmar dos factores claramente definidos de cinco ítems cada uno: Temperamento y Reacción. El factor Temperamento explica $74 \%$ de la varianza en mujeres y $60 \%$ de la variabilidad en varones. Los pesos factoriales de estas subescalas son también elevados; en el factor Temperamento estos pesos varían entre .59 y .79 en mujeres, y $.60 \mathrm{y}$ .82 en varones. Los pesos de los ítems del factor "reacción" en mujeres varían entre .48 y .75; en varones estos pesos fluctúan entre .49 y .77 (véase la tabla 2). Los coeficientes alpha de la escala de Hostilidad demuestran una elevada consistencia interna de ambos factores, correspondiendo .86 en mujeres y .82 en varones.

Tabla 2

Estructura factorial de los ítems de hostilidad del Inventario Multicultural Lsatinoamericano de la Hostilidad ( Imulho )

\begin{tabular}{|c|c|c|c|c|}
\hline \multirow[t]{2}{*}{ Ítems rasgo } & \multicolumn{2}{|c|}{ Temperamento } & \multicolumn{2}{|c|}{ Reacción } \\
\hline & Mujeres & Varones & Mujeres & Varones \\
\hline 03. Soy una persona exaltada & .79 & .82 & & \\
\hline 10. Tengo un humor colérico & .77 & .60 & & \\
\hline 02. Tengo un carácter irritable & .76 & .69 & & \\
\hline 05. Me enojo muy fácilmente & .73 & .64 & & \\
\hline 01. Soy muy temperamental & .59 & .66 & & \\
\hline 08. Me siento furioso cuando hago buen trabajo & & & .75 & .77 \\
\hline 04. Me enfado cuando hago algo bien y no es & & & .68 & .49 \\
\hline 09. Me enfado cuando alguien arruina mis planes & & & .62 & .74 \\
\hline 06. Me pone furioso que me critiquen delante de & & & .57 & .70 \\
\hline 07. Me pone furioso cometer errores estúpidos & & & 48 & .51 \\
\hline Eigenvalues & 3.96 & 3.39 & 1.01 & 1.27 \\
\hline Porcentaje de varianza & $74 \%$ & $60 \%$ & $19 \%$ & $23 \%$ \\
\hline
\end{tabular}




\section{CONCLUSIONES}

Uno de los propósitos más importantes de este trabajo fue reportar la construcción y adaptación del Inventario Multicultural Latinoamericano de la Hostilidad, que tiene como propósito evaluar la experiencia y expresión de la hostilidad en diversos países de América del Sur, México y el Caribe.

Al mismo tiempo, nos propusimos analizar la estructura factorial del instrumento. Los resultados del análisis factorial del Imulho confirman la similitud en la estructura factorial de este instrumento y el STAS, y demuestra empíricamente que la hostilidad es un concepto básicamente multidimensional y multicultural. La tabla 2 provee muy claramente evidencia empírica de la validez de construcción de la escala de Hostilidad. La solución factorial de esta escala demuestra y confirma los mismos factores encontrados en el instrumento original el STAS: Temperamento y Reacción. Los pesos factoriales de estas dos subescalas son elevadas en el factor dominante con valores insignificantes en el otro factor. Asimismo, no se observa ningún ítem con pesos dobles, lo cual demuestra evidencia de dos factores básicamente independientes. Este resultado ofrece soporte empírico al marco conceptual y teórico de la presente versión de este instrumento. El inventario, incluyendo las subescalas de Hostilidad, se presenta en el apéndice de este artículo.

\section{REFERENCIAS}

Ader, R. (Ed.) (1981). Psychoneuroimmunology. Nueva York: Academic Press.

Ader, R. (1983). Developmental psychoneuroimmunology. Developmental Psychobiology, 16, 251-267.

Ader, R. \& Cohen, N. (1985). CNSimmune system interactions: conditioning phenomena. Behavior and Brain Science, 8, 379-426.

Ader, R.; Felten, D. L. \& Cohen, N. (1990). Interactions between the brain and the immune system. Annual Review of Pharmacology and Toxicology, 30, 561-602.

Ader, R. y Cohen, N. (1991). The influence of conditioning on immune responses. En: Ader, R.; Cohen, N. \& Felten, D. L. (Eds.). Psychoneuroimmunology. Nueva York: Academic Press, 611-646.

Anson, O.; Carmel, S.; Levenson, A.; Bonneh, D. Y. \& Maoz, B. (1993). Coping with recent life events: The interplay of personal and collective resources. Behavioral Medicine, 18, 159-166.

Antoni, M. H.; Schneiderman, N.; Fletcher, M. A.; LaPerriere, A.; Goldstein, D. \& Ironson, G. (1990). Psychoneuroimmunology and HIV1. Journal of Consulting and Clinical Psychology, 58, 1, 38-49. 
Biaggio, M. K.; Supplee, K. \& Curtis, N. (1981). Reliability and validity of four anger scales. Journal of Personality Assessment, 45, 639-648.

Brislin, R. W. (1970). Back-translation for cross-cultural research. Journal of Cross-Cultural Psychology, 1, 3,185-216.

Carver, C. S.; Scheier, M. F. \& Weintraub, J. K. (1989). Assessing coping strategies: A theoretically based approach. Journal of Personality and Social Psychology, 56, 267-283.

Carver, C. S.; Pozo, C.; Harris, S. D.; Noriega, V.; Scheier, M. F.; Robinson, D. S.; Ketcham, A. S.; Moffat, Jr.; F. L. \& Clark, K. C. (1993). How coping mediates the effect of optimism on distress: A study of women with early stage breast cancer. Journal of Personality and Social Psychology, 65, 375-390.

Cohen, F.; Horowitz, M. J.; Lazarus, R. S.; Moos, R. H.; Robins, L. N. et al. (1982). Panel report on psychosocial assets and modifiers of stress. En: Cohen, M. J.; Horowitz, R. S.; Lazarus, R. H.; Moos, L. \& Robins, N. (Eds.). Stress and Human Health: Analysis and Implications of Research, 147-188.

Christensen, A. J.; Edwards, D. 1.; Wiebe, J. S.; Benotsch, E. G.; Mckelvey, L.; Andrews, M. \& Lubaroff, D. M. (1996). Effect of
Verbal Self-disclosure on natural killer cell activity: Moderating influence of cynical hostility. Psychosomatic Medicine, 58 (2): 150-155.

Dorian, B. J.; Keystone, E.; Garfinkel, P. E. \& Brown, G. M. (1982). Aberrations in lymphocyte subpopulations and functions during psychological stress. Clinical and Experimental Immunology, 50, 132-138.

Drasgow, F. \& Hulin, C. (1987). Crosscultural measurement. Interamerican Journal of Psychology, 21, 1 \& 2, 1-24.

Elliott, G. R. \& Eisdorfer, C. (Eds.). (1982). Stress and Human Health: Analysis and Implications of Research. Nueva York: Springer.

Fleming, I.; Baum, A.; Davidson, L. M.; Rectanus, E. \& McArdle, S. (1987). Chronic stress as a factor in physiologic reactivity to challenge. Health Psychology, 6, 221-237.

Glaser, R. \& Kiecolt-Glaser, J. (1987). Stress-associated depression in cellular immunity: Implications for Acquired Immune Deficiency Syndrome (AIDS). Brain, Behavior and Immunity, 1, 107-112.

Glaser, R.; Rice, J.; Sheridan, J.; Fertel, R.; Stout, J. et al. (1987). Stressrelated immune suppression: health implications. Brain, Behavior and Immunity, 1, 7-20. 
Gorsuch, R. L. (1988). Exploratory factor analysis. En: J. R. Nesselroade \& Cattell, R. B. (Eds.). Handbook of multivariate experimental psychology (2. ${ }^{\mathrm{a}}$ Ed.). Nueva York: Plenum Press, pp. 231-258.

Hulin, C. L. (1987). A psychometric theory of evaluations of item and scale translations. Journal of Cross-Cultural Psychology, 18, 2, 115-142.

Jacobs, S.; Mason, J.; Kosten, T.; Kasl, S.; Ostfeld, A. et al. (1985). Acute bereavement, threatened loss, ego defenses and adrenocortical function. Psychotherapy and Psychosomatics, 44, 151-159.

Jemmott, J. B. \& Locke, S. E. (1984). Psychosocial factors, immunologic mediation, and human susceptibility to infectious diseases: How much do we know? Psychological Bulletin, 95, 52-77.

Kiecolt-Glaser, J. \& Glaser, R. (1988a). Psychological influences in immunity: Implications for AIDS. American Psychologist, 43, 892-898.

Kemeny, M.; Zegans, L. \& Cohen, F. (1987). Stress, mood, immunity, and recurrence of genital herpes. Annual of the New York Academy of Science, 496, 735-736.

Kessler, R. C. \& Wortman, C. B. (1988). Social and psychological factors in health and illness. En: Freeman, H. E. \& Levine, E. (Eds.). Handbook of Medical Sociology. Englewood Cliffs, NJ: PrenticeHall, 403-431 (en prensa).

Kiecolt-Glaser, J. K. \& Glaser, R. (1988). Behavioral influences on immune function: evidence for the interplay between stress and health. En: Field, P. M.; McCabe, N.; Schneiderman (Eds.). Stress and Coping Across Development. Hillsdale, NJ: Lawrence Erlbaum, 96-124 (en prensa).

LaPerriere, A.; Schneiderman, N.; Antoni, M. \& Fletcher, M. (1989). Aerobic exercise training and psychoneuroimmunology in AIDS research. En: Baum, A. \& Temoshok, (Eds.). Psychosocial Perspectives on AIDS. Hillsdale, NJ.: Lawrence Erlbaum, 56-91.

Lazarus, R. S. \& Folkman, S. (1984). Stress, appraisal, and coping. Nueva York: Springer.

Matthews, K. A.; Haynes, S. G. (1986). Type A behavior pattern and coronary risk: update and critical evaluation. American Journal of Epidemiology, 123, 923-960.

Moscoso, M. S. (2000). Estructura factorial del Inventario Multicultural Latinoamericano de la Expresión de la Cólera y Hostilidad. Revista Latinoamericana de Psicología, 32, 2: 321-343. 
Moscoso, M. S. (1989). Multi-component therapy in Type A behavior pattern and coronary heart disease. Revista Intercontinental de Psicología y Educación, 2, 1 \& 2: 83-104.

Moscoso, M.S. (1992). Stress y enfermedad: Un enfoque terapéutico cognitivo a través de EMG-biofeedback-relajacion. Revista Intercontinental de Psicología y Educación, 5, 2:129-146.

Moscoso, M. S. (1995). Manejo cognitivo-conductual del stress en individuos VIH seropositivos: Revisión de media década de investigación. Avances en Psicología Clínica Latinoamericana, 13, 39-53.

Moscoso, M.S. (1997). Medición de la cólera y hostilidad: Implicaciones en el tratamiento psicológico con pacientes VIH/SIDA. Revista Peruana de Psicología, 2, 4: 39-64.

Mays, V., \& Moscoso, M. S. (1995). AIDS pandemic burdens developing world. APA Psychology International, 6, 1-7.

Moscoso, M. S., \& Pérez-Nieto, M. A. (2003). Anger, hostility and aggression assessment. En: Fernández-Ballesteros, R. (Ed.). Encyclopedia of Psychological Assessment. Londres: Sage Publications.

Moscoso, M. S. \& Spielberger, C. D. (1998). Cross-cultural assessment of the experience, expression, and control of anger. Documento pre- sentado en el 24 Congreso Internacional de Psicología. San Francisco, California, agosto.

Moscoso, M. S. \& Reheiser, E. C. (1996). The multicultural Spanish inventory of experience of anger: State and trait anger. Paper presented at the Thirteenth Congress of the International Association for CrossCultural Psychology, Montreal, agosto.

Moscoso, M. S. \& Spielberger, C. D. (1997). Measuring the experience, expression and control of anger in Latin America. Documento presentado en el Congreso Regional de Psicología para Profesionales de las Américas. Interfacing the Science and Practice of Psychology, México, DF.

Moscoso, M. S. \& Spielberger, C. D. (1999). Evaluación de la experiencia, expresión y control de la cólera en Latinoamérica. Revista de Psicología Contemporánea, 6 (1): 4-13.

Moscoso, M. S. \& Spielberger, C. D. (1999b). Measuring the experience, expression, and control of anger in Latin America: The Spanish multicultural State-Trait Anger Expression Inventory. Interamerican Journal of Psychology, 33 (2): 29-48.

Pearlin, L. I. (1991). The study of coping: An overview of problems and directions. En: Pearlin, L. I. The So- 
cial Context of Coping. Nueva York: Plenum Press, 261-275.

Peterson, C. \& Seligman, M. E. P. (1987). Explanatory style and illness. Journal of Personality, 55, 237-265.

Scheier, M. F. \& Carver, C.S. (1987). Dispositional optimism and physical well-being: the influence of generalized outcome expectancies on health. Journal of Personality, $55,169-210$.

Sechrest, L.; Fay, T. \& Hafeez Zaidi, S. (1972). Problems of translation in cross-cultural research. Interamerican Journal of Psychology, 3, 1, 41-58.

Sperber, A. D.; Devellis, R. F. \& Boehlecke, B. (1994). Cross-cultural translation. Journal of CrossCultural Psychology, 25, 4: 501-524.

Solomon, G. F. \& Temoshok, L. (1987). A psychoneuroimmunologic perspective on AIDS research: Questions, preliminary findings and suggestions. Journal of Applied and Social Psychology, 17, 3: 286-308.

Spielberger, C. D. \& Moscoso, M. S. (1995). La expresión de cólera y hostilidad y sus consecuencias en el sistema cardiovascular. Psicología Contemporánea, 2, 1: 32-43.

Spielberger, C. D., \& Moscoso, M. S. (1996). Emotional reactions to stress: Anxiety and anger. Advances in Latin American Clinical Psychology, 14, 59-82.

Spielberger, C. D.; Moscoso, M. S. \& Brunner, T. M. (2005). Cross-cultural assessment of emotional states and personality traits. En: Hambleton, R. K.; Merenda, P. F. \& Spielberger, C. D. (Eds.). Adapting educational and psychological tests for cross-cultural assessment. Hillsdale, NJ: Lawrence Erlbaum Associates.

Spielberger, C. D.; Jacobs, G.; Russell, S. \& Crane, R. (1983). Assessment of anger: The State-Trait Anger Scale. En Butcher, J. N. \& C. D. Spielberger (Eds.). Advances in personality assessment. Hillsdale, NJ: Lawrence Erlbaum Associates, 159-187.

Stoney, C. M. \& Engebretson, T. O. (2000). Plasma Homocysteine concentrations are positively associated with hostility and anger. Life Science, 66 (23): 2267-2275.

Suarez, E. C. (2004). C-Reactive protein is associated with psychological risk factor of cardiovascular disease in apparently healthy adults. Psychosomatic Medicine. 66, 684-691.

Suarez, E. C. (2003) Joint effect of hostility and severity of depressive symptoms on plasma Interleukin-6 concentration. Psychosomatic Medicine, 65, 523-527.

Suarez, E. C.; Lewis, J. G. \& Kuhn, C. (2003). Hostility raise levels of a protein associated with clogged 
arteries and greater risk for heart attack. Brain, Behavior and Immunity. Vol 16, 6.

Taylor, S. E.; Kemeny, M. E.; Aspinwall, L. G.; Schneider, S. G.; Rodríguez, R. \& Herbert, M. (1992).
Optimism, coping, psychological distress, and high-risk sexual behavior among men at risk for Acquired Immunodeficiency Syndrome (AIDS). Journal of Personality and Social Psychology, 63, 460-473.

\section{APÉNDICE}

Inventario Multicultural Latinoamericano de la Hostilidad

Edad

Sexo

Nacionalidad

Profesión

\section{INSTRUCCIONES}

PARTE 1: A continuación se dan una serie de expresiones que la gente usa para describirse a sí mismos. Lea cada frase y marque el número apropiado en la sección de respuestas que indique cómo se siente generalmente.

$$
1=\text { Casi nunca } \quad 2 \text { = Algunas veces } \quad 3 \text { = Frecuentemente } \quad 4=\text { Casi siempre }
$$

01. Soy muy temperamental.

02. Tengo un carácter irritable.

03. Soy una persona exaltada

04. Me enfado cuando hago algo bien y no es apreciado.

05. Me enojo muy fácilmente.

06. Me pone furioso (a) que me critiquen delante de los demás.

07. Me pone furioso (a) cuando cometo errores estúpidos.

08. Me siento furioso cuando hago un buen trabajo y se me valora poco.

09. Me enfado cuando alguien arruina mis planes

10. Tengo un humor colérico.

$\begin{array}{llll}1 & 2 & 3 & 4 \\ 1 & 2 & 3 & 4 \\ 1 & 2 & 3 & 4 \\ 1 & 2 & 3 & 4 \\ 1 & 2 & 3 & 4 \\ 1 & 2 & 3 & 4 \\ 1 & 2 & 3 & 4 \\ 1 & 2 & 3 & 4 \\ 1 & 2 & 3 & 4 \\ 1 & 2 & 3 & 4\end{array}$

